\title{
DUALITY AND KATO'S THEOREM ON SMALL PERTURBATIONS
}

\author{
BY LE QUANG CHU
}

Communicated by Chandler Davis, July 6, 1976

\begin{abstract}
The main result is a theorem on stability of index under small perturbations in locally convex spaces, which reduces for Banach spaces to the familiar theorem of T. Kato.
\end{abstract}

It is a well-known result of Gohberg and Krein [2] and Kato [3] that if $T$ is a semi-Fredholm operator and $P$ a bounded operator of norm small enough, then $T+P$ is a semi-Fredholm operator with the same index as $T$. Kato gives a precise upper bound of the norm of $P$ : $\|P\|<\gamma(T)$, where $\gamma(T)$ essentially is $\left\|\hat{T}^{-1}\right\|^{-1}, \hat{T}$ being the one-to-one operator induced by $T$. In geometric terms, this may be expressed as $T B \supset \lambda B^{\prime} \cap R(T), P B \subset \mu B^{\prime}$ and $0 \leqslant \mu<\lambda, B, B^{\prime}$ being the unit balls of $E, F$.

Some results concerning small bounded perturbations of $\Phi_{-}$-operators in more general locally convex spaces are given in [7], [4], but they do not fully render the precise Kato theorem in case of Banach spaces.

By using Kato's theorem in the dual, we obtain some results (Propositions 1 and 3) which do constitute an extension of Kato's theorem on small perturbations of $\Phi_{-}$-operators, and refine several results in [7] , [4] .

In the sequel, $E, F$ always denote two Hausdorff locally convex spaces, and $T, P$ two (linear) operators from $E$ into $F$ such that $[D(T)]^{-} \subset D(P),[D(T)]^{-}$ being the closure of the domain of $T$. Let $N(T)$ and $R(T)$ denote the kernel and the range of $T$. By neighborhood we mean an absolutely convex neighborhood of the origin. A disk is an absolutely convex set.

The operator $T$ is open (resp. almost open) if $T U$ (resp. [TU $]^{-}$) is a neighborhood in $R(T)$, for any neighborhood $U \subset E . \quad T$ is a $\Phi_{-}$(resp. $\Phi_{+}$)operator if $T$ is open, has a closed graph (in $E \times F$ ) and a closed range, and codim $R(T)<\infty$ (resp. $\operatorname{dim} N(T)<\infty)$. The index of $T$ is then defined as $\operatorname{ind}(T)=\operatorname{dim} N(T)-\operatorname{codim} R(T)$ (we do not distinguish between different cardinalities of infinity).

Proposition 1. Let $T$ be an almost open operator with $\operatorname{codim}[R(T)]^{-}$ $<\infty$, and $P$ a continuous operator.

(1) Assume that there exists a base of neighborhoods $U$ in $E$ such that

AMS (MOS) subject classifications (1970). Primary 47A55, 47B30. 
$P U \cap[R(T)]^{-} \subset \epsilon[T U]^{-}, 0<\epsilon<1$.

(2) Assume further that for some neighborhood $U_{0} \subset E$, there are bounded disks $B, B^{\prime}$ such that $P U_{0} \subset[T B]^{-}+B^{\prime}$.

Then $T+P$ is almost open and $\operatorname{codim}[R(T+P)]^{-} \leqslant \operatorname{codim}[R(T)]^{-}$. If $E$ is a Fréchet space, or, more generally, fully complete [6], and $T$ has a closed graph, then $T$ and $T+P$ are $\Phi_{-}-$operators and $\operatorname{ind}(T+P)=\operatorname{ind}(T)$.

Corollary 2. Conditions (1) and (2) are satisfied, and Proposition 1 holds true if there exist a neighborhood $U_{0}$, a bounded disk $B$ and $0<\epsilon<1$ such that $B \subset \epsilon U_{0}, P U_{0} \cap[R(T)]^{-} \subset[T B]^{-}$and $P U_{0}$ is bounded.

The proof of Proposition 1 uses duality and Kato's theorem applied to the Banach spaces generated by closed equicontinuous sets, and is very much similar to those in [4]. Some technical modifications lead to the following more general formulation:

Proposition 3. Let $T$ be an almost open operator with $\operatorname{codim}[R(T)]^{-}$ $<\infty$ and $P$ a continuous operator.

(1') Assume that there is a base of neighborhoods $U$ in $E$ such that for any $U \in U$, there exist a finite-dimensional subspace $N \subset[R(T)]^{-}$, and $0<\epsilon<1$, for which $P U \cap[R(T)]^{-} \subset \epsilon[T U]^{-}+N$.

(2') Assume further that for some neighborhood $U_{0} \subset E$, a finitedimensional subspace $N_{0}$, and bounded disks $B, B^{\prime}, P U_{0} \subset[T B]^{-}+B^{\prime}+N_{0}$.

Then $T+P$ is almost open and

$$
\operatorname{codim}[R(T+P)]^{-} \leqslant \operatorname{codim}[R(T)]^{-}+\operatorname{dim}\left(N+N_{0}\right)<\infty .
$$

If $E$ is fully complete, and $T$ has a closed graph, then $T+P$ and $T$ are $\Phi \_$operators and $\operatorname{ind}(T+P)^{\prime}=\operatorname{ind}(T)$.

Corollary 4. Condition $\left(1^{\prime}\right)$ is satisfied if there exist a neighborhood $U \subset E$, a bounded disk $B_{0}$, a precompact disk $K$, a finite-dimensional subspace $N^{\prime}$ and $0<\epsilon<1$ such that $B_{0} \subset \epsilon U$ and $P U \cap[R(T)]^{-} \subset\left[T B_{0}\right]^{-}+K+N^{\prime}$. $K+N^{\prime}$.

Both conditions $\left(1^{\prime}\right)$ and $\left(2^{\prime}\right)$ are satisfied in particular if $P U \subset\left[T B_{0}\right]^{-}+$

$$
\text { If } P U \subset\left[T B_{0}\right]^{-}+N^{\prime} \text { then }[R(T+P)]^{-}+N^{\prime}=[R(T)]^{-}+N^{\prime} \text {. }
$$

Remarks, Again, by application of a result of Kato in the dual, it could be shown that $\operatorname{codim}[R(T+\lambda P)]^{-}$is constant for $|\lambda| \neq 0$ and small enough.

Corollary 4 yields at the same time Theorem $4 . \mathrm{b}$, and the remarks following Theorems 2 and 4 in [7], where the perturbations are of the type $P U \subset\left[T B_{0}\right]^{-}+N^{\prime}$ and $P U \subset\left[T B_{0}\right]^{-}+K$ (K compact). It also provides another short proof of the main part of Theorem 2 in [8] (see also [5]), and shows that precompact perturbations of $\Phi_{-}$-operators may be reduced to small perturbations. 
We would like also to point out that.duality is a convenient tool to study the stability of "almost-openness" of $\Phi_{+}$and $\Phi_{-}$-operators under small or precompact perturbations. The stability of the index is readily obtained when suitable assumptions of completeness are placed on the spaces in such a way that the perturbed operator becomes a $\Phi_{+}$or $\Phi_{-}$-operator.

\title{
REFERENCES
}

1. M. De Wilde and Le Quang Chu, Perturbation of maps in locally convex spaces, Math. Ann. 215 (1975), 215-233.

2. I. C. Gohberg and M. G. Kreĭn, The basic propositions on defect humbers, root numbers and indices of linear operators, Uspehi Mat. Nauk 12 (1957), no. 2 (74), 43-118; English transl., Amer. Math. Soc. Transl. (2) 13 (1960), 185-264. MR 20 \#3459; 22 \#3984.

3. T. Kato, Perturbation theory for nullity, deficiency and other quantities of linear operators, J. Analyse Math. 6 (1958), 261-322. MR 21 \#6541.

4. Le Quang $\mathrm{Chu}$, Bounded perturbations of $\Phi_{+}$and $\Phi_{-}$-operators in locally convex spaces, Bull. Soc. Roy. Sci. Liège 1-2 (1975), 28-35; addendum, ibid. 9-10 (1975), 537-539; corrigendum, ibid. (to appear).

5. - A short proof of Vladimirskii's theorem on precompact perturbations, Canad. Math. Bull. 18 (1975), 649-655.

6. A. P. Robertson and W. Robertson, Topological vector spaces, 2nd ed., Cambridge Univ. Press, New York, 1973. MR 50 \#2854.

7. Ju. N. Vladimirskir, On bounded perturbations of $\Phi$-operators in locally convex spaces, Dokl. Akad. Nauk SSSR 196 (1971), 263-265 = Soviet Math. Dok1. 12 (1971), 80-83. MR 42 \#8335.

8. Compact perturbations of $\Phi$-operators in locally convex spaces, Sibirsk. Mat. Ž. 14 (1973), 738-759 = Siberian Math. J. 14 (1973), 511-524.

\author{
ONTARIO, CANADA
}

DEPARTMENT OF MATHEMATICS, MCMASTER UNIVERSITY, HAMILTON, 